\title{
EL CASO LAGO AGRIO: MULTIPOLARIDAD EN LA LITIGACIÓN CIVIL INTERNACIONAL
}

\author{
MARTA REQUEJO ISIDRO \\ Senior Research Fellow Max Planck Institute Luxembourg \\ marta.requejo@mpi.lu
}

Recibido: 15 de mayo de 2014 / Aceptado: 5 de junio de 2014

RESUMEN: La "litigación de Lago Agrio" ejemplifica la fragmentación de la tutela efectiva de los particulares en el ámbito de la litigación civil transnacional por daños medioambientales. Más de cinco jurisdicciones nacionales se han visto implicadas hasta ahora en el supuesto: unas en virtud de una solicitud de tutela declarativa, otras por la petición de exequátur de la decisión ecuatoriana en otros Estados. Además, las partes (a veces una, a veces otra) han puesto en marcha tres vías distintas de resolución de controversias: la judicial ante los tribunales domésticos, la arbitral en el marco del arbitraje de inversiones y una internacional destinada a la protección de los derechos fundamentales de los ciudadanos frente a los Estados. Un escenario tan complejo da pie a numerosas preguntas. El presente trabajo surge de la duda sobre el funcionamiento armónico de las múltiples instancias accesibles a los diferentes actores, abordando sin pretensión de exhaustividad la cuestión del "reparto" de causas y argumentos ante ellas.

RESUM: La "litigació de Lago Agrio" exemplifica la fragmentació de la tutela efectiva dels particulars en l'àmbit de la litigació civil transnacional per danys mediambientals. Més de cinc jurisdiccions nacionals s'han vist implicades fins ara en el supòsit: unes en virtut d'una sol·licitud de tutela declarativa, altres per la petició d'exequàtur de la decisió equatoriana en altres estats. A més, les parts (de vegades una, de vegades una altra) han posat en marxa tres vies diferents de resolució de controvèrsies: la judicial davant els tribunals domèstics, la arbitral en el marc de l'arbitratge d'inversions i una internacional destinada a la protecció dels drets fonamentals dels ciutadans enfront dels Estats. Un escenari tan complex dóna peu a nombroses preguntes. Aquest treball sorgeix del dubte sobre el funcionament harmònic de les 
múltiples instàncies accessibles als diferents actors, abordant sense pretensió d'exhaustivitat la qüestió del "repartiment" de causes i arguments davant d'elles.

ABSTRACT: The "Lago Agrio litigation" exemplifies the fragmentation of the effective protection of rights in the field of transnational civil litigation for environmental damage. So far, over five national jurisdictions have been involved: some under a request for a declaratory judgment, others by a request for the exequatur of the Ecuadorian ruling in other fora. In addition parties have engaged in three different ways of resolution of disputes: judicial, before the domestic courts of several countries; arbitral, under investment arbitration; and international, before institutions in charge of the protection of the fundamental rights of citizens against the State. Such a complex scenario gives rise to many questions. This paper takes as departure point the general question about how multiple mechanisms of dispute resolution can work simultaneously and smoothly; with no claim to completeness, it addresses the issue of where (in which jurisdiction or instance) the different actors should be allowed to raise -or, as the case may be, should better raise- their arguments.

PALABRAS CLAVE: Responsabilidad civil - Chevron Corp. - Tutela judicial Exequátur - Ejecución - Arbitraje de inversiones

PARAULES CLAU: responsabilitat civil — Chevron Corp. — Tutela judicial — Exequàtur — Execució - Arbitratge d'inversions

KEY WORDS: Civil liability - Chevron Corp. - Due process - Exequatur Enforcement — Investment arbitration

Sumario: I. Introducción. II. El supuesto. 1. Hechos. 2. La fase de tutela declarativa: de EE. UU. a Ecuador. 3. Actuaciones paralelas. III. La victoria que no es tal. 1. La ejecución de la sentencia, o historia de un peregrinaje inacabado. 1.1. Excurso: por qué no en EE. UU. 1.2. En Canadá. 1.3. En Brasil. 1.4. En Argentina. 2. El arbitraje de inversiones. 2.1. Las órdenes interinas "antiproceso". 2.2. La reacción de los particulares afectados. 2.3. Primer laudo arbitral parcial, track I. IV. El reparto de asuntos, o dónde se discute qué. 1. El argumento del fraude procesal: en origen o ( $\mathrm{y}$ ?) en el Estado requerido. 2. Inversiones extranjeras y los derechos de terceros: ¿arbitraje o tutela judicial? 3. La falta de legitimación pasiva/falta de bienes: en exequátur o en fase de ejecución. V. Conclusión. VI. Bibliografía. 


\section{INTRODUCCIÓN}

El complejo entramado de actuaciones que se conoce como "litigación de Lago Agrio"1 es un ejemplo perfecto para los académicos en el ámbito de la litigación civil transnacional por violaciones medioambientales. Hasta el momento más de cinco jurisdicciones nacionales se han visto implicadas, bien porque se ha solicitado de ellas tutela declarativa, bien porque se les ha pedido tutela en forma de reconocimiento de la decisión pronunciada en otro lugar. Las partes (a veces una, a veces otra) han recurrido a tres mecanismos distintos de resolución de controversias: judicial, arbitral e internacional. Por todo ello, el supuesto ejemplifica como ningún otro la fragmentación de la tutela judicial efectiva de los particulares en el escenario global. Al mismo tiempo pone de manifiesto lo artificial del "public/private divide" en el derecho procesal y sustantivo actuales; sirve también para resaltar las tensiones entre la protección de los inversores y otras obligaciones de los Estados; y ratifica la dificultad de articular procedimientos arbitrales y judiciales ${ }^{2}$. En la práctica representa lo que se ha dado en llamar "multipolaridad de la litigación transnacional"3 y revela la utilización por los particulares de diferentes instrumentos procesales y procedimentales concebidos para la defensa de derechos legítimos en formas estratégicas, tal vez no tan legítimas como aquellos derechos.

El epígrafe II, esencialmente descriptivo, es instrumental respecto a los que siguen y sirve para introducir el caso ${ }^{4}$. Bajo el número III abordamos puntos clave del desarrollo reciente del supuesto. En el IV analizamos algunos aspectos seleccionados que hemos elegido a partir de formularnos la pregunta sobre el reparto de cuestiones entre las diversas jurisdicciones

\footnotetext{
${ }^{1}$ Este trabajo continúa una serie de artículos publicados en torno al caso Lago Agrio a medida que este ha ido avanzando. Vid. REQUEJO ISIDRO, M., “Aguinda v. Texaco: de EEUU a Ecuador, y vuelta (¿a ninguna parte?)", Fernández Albor, A. y Pérez Carrillo, E. (eds.), Empresa Responsable y Crecimiento Sostenible: Aspectos Conceptuales, Societarios y Financieros, Thomson Reuter, 2012; id., "Aguinda v. Texaco en la fase de exequatur: análisis del primer tropiezo", Dereito, Revista Xurídica da Universidade de Santiago de Compostela, 2013; id., "The Last Struggle for Redress: Recognition and Enforcement of Foreign Resolutions on Civil Liability for Human Rights Violations", Companies in Conflict Situations, Generalitat de Catalunya/ICIP, 2013. Por su parte, la RCDA dedicó en 2013 un editorial a Chevron Corp. y su actitud en este litigio bajo el título "The Procrastinator. Chevron a l'Ecuador".

2 STEINHARDT, R. G., “Remarks”, 106 Am. Soc'y Int'l L. Proc. 422 (2012).

${ }^{3}$ WHYTOCK, C. A., "The Chevron-Ecuador Case: Three Dimensions of Complexity in Transnational Dispute Resolution", 106 Am. Soc'y Int'l L. Proc. 425 (2012); id., "Some Cautionary Notes on the Chevronization of Transnational Litigation”, 1 Stan. J. Complex Litig. 467 (2012-2013), pp. 468-469.

${ }^{4}$ Las decisiones de los diferentes tribunales que van marcando las etapas del caso son (casi en su totalidad) $\begin{array}{lllll}\text { accesibles la } & \text { desde } & \text { la } & \text { web } & \text { http://www.business- }\end{array}$ humanrights.org/Categories/Lawlawsuits/Lawsuitsregulatoryaction/LawsuitsSelectedcases/TexacoChevronlaws uitsreEcuador $>$.
} 
implicadas (simplificando: dónde se discute qué) en un escenario tan complejo como el que han creado las partes en la litigación de Lago Agrio. El apartado V cierra con conclusiones.

\section{EL SUPUESTO ${ }^{5}$}

\section{Hechos}

En 1993 un grupo de nacionales de Ecuador planteó demanda en Nueva York en forma de class action contra la compañía energética Texaco, alegando graves abusos medioambientales en la región amazónica de Oriente; reclamaban compensación por los daños y perjuicios resultantes de la contaminación de los ríos y los bosques de lluvia durante las operaciones de perforación y explotación de petróleo en la zona por parte de la compañía.

La relación de Texaco con Ecuador se remonta a hace más de treinta años. En la década de 1960 el Gobierno ecuatoriano invitó a dos compañías, Texaco y Gulf Oil Corporation, a realizar prospecciones en busca de petróleo en Oriente. Las sociedades formaron un consorcio en el que luego, en la época de la dictadura militar, entró a formar parte el Gobierno ecuatoriano. En la década de 1980 el petróleo se situó en la base de los ingresos de Ecuador, si bien decayó posteriormente al declinar la producción. El espectacular crecimiento de la industria del petróleo se produjo a costa de daños medioambientales con repercusiones en la vida y la salud humanas: los vertidos de crudo y de productos relacionados en la cuenca del Amazonas se calculan en millones de toneladas. Un estudio de 1993 estimó que los residentes de Oriente estaban expuestos a niveles de contaminación relacionados con el petróleo por encima de los estándares admitidos internacionalmente; también puso de manifiesto elevadas tasas de enfermedades dermatológicas entre ellos. Más adelante la investigación relacionó tales descubrimientos con incrementos en el riesgo de padecer enfermedades reproductivas y neurológicas, así como cáncer. Pruebas aportadas en el juicio demostraron que Texaco trató los residuos conforme a técnicas que en EE. UU. habían sido abandonadas por otros operadores como consecuencia del incremento de la preocupación por la salud y el impacto ambiental.

Texaco dejó de operar en Ecuador en 1992. En marzo de 1995 firmó con el Gobierno del país un acuerdo sobre reparaciones medioambientales en virtud del cual la empresa se comprometía a invertir 40 millones de dólares en la limpieza de la zona a cambio de la

\footnotetext{
${ }^{5}$ Retomamos la descripción que hicimos en trabajos previos (vid. supra nota al pie núm. 1) con la pertinente actualización.
} 
renuncia a toda demanda futura. En 2001 Texaco fue adquirida por Chevron Corp., la segunda compañía energética más importante del mundo.

\section{La fase de tutela declarativa: de EE. UU. a Ecuador}

La primera demanda de las comunidades afectadas en Ecuador data de noviembre de 1993. La acción se interpuso ante un tribunal federal del Distrito Sur de Nueva York, al ser esta ciudad la sede de la administración central de las operaciones internacionales de la empresa y donde, por lo tanto, se habrían adoptado las decisiones concernientes a la explotación de crudo en Ecuador. El demandado contestó la competencia de los tribunales de EE. UU. alegando que, al amparo de la doctrina de forum non conveniens, Ecuador sería un foro más apropiado. Tras nueve años el tribunal consideró Ecuador como un foro alternativo adecuado a pesar de los argumentos de la parte demandante sobre la parcialidad y corrupción de la justicia local. En contrapartida, el demandado tuvo que comprometerse a aceptar cualquier decisión que recayera en Ecuador, dentro de los límites establecidos por la Uniform Foreign Money Judgments Recognition Act (UFMJRA) ${ }^{6}$.

Contra todo pronóstico, el caso fue retomado en Ecuador en 2003 ante la Corte Provincial de Sucumbíos. En febrero de 2011 este tribunal dictó una sentencia de casi 200 páginas (la decisión de Lago Agrio) en la cual afirmaba haber encontrado evidencias incontestables del vertido deliberado, entre 1964 y 1993, de toneladas de residuos tóxicos en la zona considerada y de daños al medio ambiente y la salud, consecuencia de dicho vertido, con un impacto tal sobre el ecosistema que está en juego la propia subsistencia de la comunidad afectada. Sobre la base de reflexiones relativas al levantamiento del velo, la decisión descartaba las alegaciones de Chevron Corp. a propósito de su relación con Texaco. El Tribunal pronunció una condena de 18 billones de dólares, de los cuales la mitad son en concepto de daños punitivos.

La apelación de Chevron Corp. fue desestimada en enero de 2012; la segunda instancia confirmó la decisión en su totalidad. En casación Chevron Corp. tuvo mejor suerte: en noviembre de 2013 la Corte Suprema reafirmaba la decisión en cuanto a los daños

\footnotetext{
${ }^{6}$ Sobre estos, vid. el apartado III, bajo 1.1.
} 
compensatorios y las costas por una suma de 9,51 billones de dólares, pero, en cambio, aceptaba el recurso en lo relativo a los daños punitivos ${ }^{7}$.

La interposición del recurso ante el Tribunal Supremo de Ecuador no suspendió el carácter ejecutivo de la decisión de instancia: con fecha de 15 de octubre de 2012 la Corte Provincial de Justicia de Sucumbíos dictó providencia por la que se decretaba el embargo de la totalidad del patrimonio de Chevron Corp. dentro y fuera del país a fin de asegurar la ejecución de la condena. Poco después los demandantes solicitaron en varios foros el reconocimiento y la declaración de ejecutividad y/o medidas relacionadas con el fin último —esto es, la ejecución de la sentencia ${ }^{8}$ -

\section{Actuaciones paralelas}

En paralelo al proceso judicial de Ecuador tenían lugar otras actuaciones relacionadas con la controversia. En 2009, durante la pendencia del proceso judicial, Chevron Corp. inició actuaciones contra el Gobierno ecuatoriano ante la Corte Permanente de Arbitraje de La Haya al amparo del Bilateral Investment Treaty (BIT) entre EE. UU. y Ecuador, en vigor desde 1997. El Tribunal Arbitral ha pronunciado medidas cautelares contra Ecuador. Por su parte, los particulares demandantes en el litigio de Lago Agrio han reaccionado pidiendo a la Comisión Interamericana de Derechos Humanos la adopción de medidas que aseguren que el Gobierno de Ecuador no interferirá en la ejecución de la sentencia judicial y, también, la suspensión del procedimiento arbitral ${ }^{9}$.

Dos semanas antes de que recayera sentencia en Ecuador, Chevron Corp. inició un proceso judicial en EE. UU. contra asociados de la parte demandante en aquel proceso al amparo de la Racketeer Influenced and Corrupt Organizations Act (RICO). En el marco de este procedimiento, la compañía solicitó una medida preventiva tendente a impedir el reconocimiento y la ejecución de la decisión obtenida en el extranjero, de ahí que sea conocida como "anti-enforcement injunction". La medida fue otorgada en primera instancia

\footnotetext{
${ }^{7}$ Con toda seguridad, de haberse conservado esta parte de la decisión, esta habría suscitado un interesante debate en sede de reconocimiento y exequátur. Desde hace años se discute la contrariedad de los daños punitivos y el orden público en aquellos países que, como el nuestro y otros muchos, consideran función esencial (si no única) de la responsabilidad civil la de compensar; la respuesta mayoritaria es la afirmativa. La pregunta es si se mantendría la misma tendencia en relación con un bien público como es el medio ambiente; también, habida cuenta de la magnitud del daño.

${ }^{8}$ Infra, bajo III.

${ }^{9}$ Infra, bajo III, apartado 2.2 .
} 
en marzo de 2011, dirigida a los demandantes en Ecuador, sus abogados y cualquier otra persona en concierto con ellos, proscribiendo "funding, commencing, prosecuting, advancing in any way, or receiving benefit from, directly or indirectly, any action or proceeding for recognition or enforcement of any judgment against Chevron [in the Ecuadorian action], or for prejudgment seizure [...]". Su alcance se pretendía mundial, lo que explica el rechazo que ha suscitado en la doctrina ${ }^{10}$. Apelada, la orden fue revocada por el siguiente motivo: la ley aplicable - in casu, la Foreign Money Judgments Recognition Act de Nueva York- no autoriza la declaración como no ejecutable de una resolución extranjera con carácter preventivo, esto es, sobre la base de una condena hipotética ${ }^{11}$.

La demanda bajo la Racketeer Influenced and Corrupt Organizations Act (RICO) ha pervivido; el 4 de marzo de 2014, la District Court del Distrito Sur de Nueva York se pronunció en el sentido de que la decisión ecuatoriana es el producto de una actividad fraudulenta e ilegal, por lo que no es susceptible de ejecución. En una orden separada el juez prohibió a los demandantes cualquier intento de ejecutar la decisión de Lago Agrio ante cualquier tribunal de EE. UU.

\section{LA VICTORIA QUE NO ES TAL}

\section{La ejecución de la sentencia, o historia de un peregrinaje inacabado}

A primera vista la decisión de Lago Agrio se parece a una victoria, tanto más valiosa cuanto que se produce en Ecuador. El propio Estado afectado toma las riendas en un escenario - el de los abusos cometidos por los inversores extranjeros en el territorio- donde lo corriente es lo contrario. No es solo una victoria judicial de unos demandantes concretos: es moral y alcanza a muchos.

Sin embargo, Chevron Corp. carece de patrimonio ejecutable en Ecuador. Oír esto puede resultar sorprendente. La realidad, sin embargo, es que esta es una de las posibilidades que la globalización ofrece a las empresas: la reestructuración corporativa, las modificaciones en la titularidad de los elementos del grupo, el desplazamiento de activos a filiales existentes o

\footnotetext{
${ }^{10}$ Vid. GÓMEZ, M.A., "The Global Chase: Seeking the Recognition and Enforcement of the Lago Agrio Judgment Outside of Ecuador", 1 Stan. J. Complex. Litig. 429 (2012-2013), p. 442 y referencias en la nota al pie núm. 81 .

${ }^{11}$ Chevron Corp. v. Donziger, 768 F. Supp. 2d 581 (SDNY 2011), otorgando la medida; Chevron Corp. v. Naranjo, 667 F. 3d 232 (2d Cir. 2012), revocándola.
} 
nuevas..., son instrumentos que permiten — entre otros fines - eludir una responsabilidad, aun declarada por sentencia ${ }^{12}$.

Así las cosas, la ejecución de la decisión de Lago Agrio deberá ser procurada (de hecho, lo está siendo) en otra u otras jurisdicciones. Hay datos para un pronóstico sobre cuáles serán estos lugares. De entrada, en la Providencia de octubre de 2012 el propio tribunal de Sucumbíos estableció una relación de más de 71 filiales de Chevron Corp. situadas en distintos continentes, apoyándose en el informe anual que Chevron Corp. misma había presentado a la Securities and Exchange Commission de EE. UU. Por otro lado, existe un informe preparado para los demandantes por el bufete Patton Boggs en el contexto de la búsqueda de third-party funding en el que se diseñaba un plan estratégico multinacional con vistas a la ejecución de la decisión ecuatoriana a favor de los damnificados ${ }^{13}$.

El propio año 2012 los demandantes solicitaron el reconocimiento y la ejecución de la sentencia en varios lugares: en Ontario (Canadá), ante la Superior Court of Justice; en Brasil, ante el Tribunal Superior de Justicia de Brasilia; y en Argentina. Se ha obtenido también una declaración de embargo de los bienes de la empresa en Colombia.

\subsection{Excurso: por qué no en EE. UU.}

La sede social de Chevron Corp. es Delaware; su lugar de actividad principal es California; el caso estuvo pendiente en Nueva York durante nueve años. La declaración de forum non conveniens del tribunal de este lugar, a la que hemos aludido en el apartado anterior, iba acompañada del compromiso de Chevron Corp. de acatar cualquier decisión de Ecuador en los límites previstos por la UFMJRA. Y, sin embargo, hasta ahora no se ha intentado el exequátur de la decisión ecuatoriana en EE. UU.

Recaída la sentencia de Lago Agrio, Chevron Corp. prometió públicamente a sus accionistas que no satisfaría ninguna condena impuesta por Ecuador: de este modo aseguraba años de

\footnotetext{
12 Vid. el análisis de HANSEN, R.F., "Multinational Enterprise Pursuit of Minimized Liability: Law, International Business Theory and the Prestige Oil Spill”, 26 Berkeley J. Int'L L. 410, al hilo del célebre caso Prestige.

${ }^{13}$ GÓMEZ, M. A., "The Global...", cit., texto nota al pie núm. 155. Esta forma de proceder, solicitando el reconocimiento en múltiples foros al mismo tiempo, ha sido calificada de "acoso" contra el demandado: SEIDERMAN, E., "The Recognition Act, Anti-Suit Injunctions, the DJA, and Much More Fun: The Story of the Chevron-Ecuador Litigation and the Resulting Problems of Aggressive Mutinational Enforcement Proceedings", 41 Fordham Urb. L.J. 265 (2013), p. 302. En realidad una y otra parte juegan con los instrumentos ofrecidos por los diversos ordenamientos: ambas en el límite del abuso y, con bastante probabilidad, ambas más allá.
} 
apelaciones en EE. UU. ${ }^{14}$. De facto, esta declaración posee un importante efecto disuasorio sobre los acreedores ecuatorianos de Chevron Corp. El último hito en la saga, la decisión de marzo de 2014 al amparo de la ley RICO que hemos citado, viene a reforzarlo.

Lo anterior no ha impedido a la doctrina preguntarse por las posibilidades de reconocimiento/exequátur en EE. UU. de un pronunciamiento contrario a los intereses de Chevron Corp. a la luz de la UFMJRA; ello, tanto antes como después de recaída la sentencia ecuatoriana $^{15}$.

De acuerdo con la UFMJRA, la ejecución de una decisión extranjera en EE. UU. requiere su previo reconocimiento. A este efecto, corresponde a quien lo solicita probar que la resolución es definitiva, determinante de derechos y deberes entre las partes y ejecutiva en el país de origen. A partir de ahí recae sobre el demandado establecer la concurrencia de alguno de los motivos de no reconocimiento, que se centran en las siguientes circunstancias: la decisión extranjera ha sido pronunciada por un sistema que no provee jueces imparciales; el tribunal de origen carecía de competencia sobre el demandado o por razón de la materia; la falta de notificación al demandado; el fraude en el proceso; la contrariedad de la demanda con el orden público del foro; la inconciliabilidad entre la decisión extranjera y otra del foro; la contrariedad entre la decisión y un acuerdo entre las partes; que la competencia del juez de origen se basara solo en la notificación al demandado, careciendo por lo demás el tribunal extranjero de contactos relevantes con el caso; que la resolución fuera pronunciada en circunstancias tales que cabe dudar de la integridad del tribunal respecto a ella; o que el proceso concreto no hubiera respetado los requisitos propios del due process.

En el contexto descrito los autores coinciden en que la excepción de fraude, ligada al requerimiento de due process y a la exigencia de un foro imparcial, son las grandes armas de Chevron Corp. a la hora de combatir el exequátur de la decisión ecuatoriana en EE. UU. ${ }^{16}$. La acusación de fraude en forma de influencia sobre el juez en cuanto al nombramiento de uno

\footnotetext{
${ }^{14}$ CASSELMEN, B., "Chevron Expects to Fight Ecuador Lawsuit in US”, Wall St. J., 20 de julio de 2009 , apartado B3.

${ }^{15}$ Antes: DHOOGE L. J., “Aguinda v. Chevron Texaco: Mandatory Grounds for the Non-recognition of Foreign Judgments for Environmental Injury in the United States”, 19 J. Transnat'l L. \& Pol'y 1, 13 (2009-2010); ídem, "Aguinda v. Chevron Texaco: Discretionary Grounds for the Non-recognition of Foreign Judgments for Environmental Injury in the United States", 28 Va. Envtl. L.J. 241 (2010). Con posterioridad, vid. WHYTOCK C.A., "Some Cautionary Notes ...”, 1 Stan. J. Complex Litig. 467; SEIDERMAN, E., "The Recognition..." cit.

${ }^{16}$ Vid. resumen en REQUEJO ISIDRO, M., “Aguinda v. Texaco: de EEUU a Ecuador...”, apartado núm. IV 3. Se descarta, en cambio, la contrariedad al orden público de la decisión por razones de fondo (esto es, no procesales): PATEL S., "Delayed Justice: A Case Study of Texaco and the Republic of Ecuador's Operations, Harms, and Possible Redress in the Ecuadorian Amazon", 26 Tul. Envtl. L.J. 71 (2012), p. 96.
} 
de los peritos y las dudas sobre la autoría intelectual de los informes de expertos e incluso de la decisión misma han sido, de hecho, esgrimidas por Chevron Corp. en otros escenarios: en su defensa en Ecuador, en el procedimiento arbitral contra la República al amparo del BIT y en Canadá y en Brasil frente a la solicitud de exequátur. La declaración jurada de uno de los jueces ecuatorianos y varias cintas de vídeo grabadas en secreto apuntan a la corrupción judicial y vienen a sostener las más que esperables alegaciones de Chevron Corp. en el sentido de que la decisión ha sido dictada en el marco de un sistema que no provee tribunales imparciales ni procedimientos compatibles con los requisitos de la tutela efectiva ${ }^{17}$.

\subsection{En Canadá}

En 2012 los demandantes en Lago Agrio iniciaron un procedimiento en Ontario para el reconocimiento y la ejecución de la decisión de Lago Agrio. Esta solicitud, cuya resolución definitiva aún está pendiente, ha sido objeto de un análisis detallado en primera instancia y en apelación. Dado que los argumentos de Chevron Corp. se reiteran en otros foros, está justificado prestarle atención en primer lugar.

La petición de homologación se dirigió contra Chevron Corp. y dos filiales canadienses de esta (Chevron Canada Limited y Chevron Finance Canada Limited ${ }^{18}$ ) que, a juicio de los demandantes, son en su totalidad propiedad de Chevron Corp. y sobre las cuales esta ejerce un control absoluto. En su defensa, Chevron Corp. alegó la falta de cualquier contacto entre ella y Ontario (ausencia de residencia, de actividad de negocio, de patrimonio) y que las afirmaciones acerca de sus vínculos con las otras compañías eran falsas. Estas últimas argumentaron la limitación del proceso y de la resolución de Ecuador a la empresa americana.

El análisis del tribunal de Ontario se centró en dos puntos: la competencia para pronunciarse sobre la solicitud de reconocimiento y exequátur — en otras palabras, la necesidad o no de que exista "some real or substantial connection to Ontario" del demandado para aceptar asumir jurisdicción sobre el caso-, y el efecto práctico que tendría pronunciarse a favor de los solicitantes de reconocimiento/exequátur ${ }^{19}$. Tras rechazar lo primero por falta de evidencias concluyentes, el tribunal canadiense examinó la relación entre las personas contra

\footnotetext{
${ }^{17}$ Se impone una precisión en este punto: en este trabajo no se afirma ni se niega que haya habido corrupción en el juicio en Ecuador ni tampoco se pretende alegar ni a favor ni en contra de las virtudes o defectos del sistema judicial ecuatoriano. Todo ello forma parte de los argumentos de Chevron, que ha logrado imponer su fuerza de convicción en determinados foros; por ese motivo, y en esa medida, deben ser incorporados al análisis doctrinal. ${ }^{18}$ Posteriormente se abandonó la acción en lo relativo a Chevron Finance Canada Limited.

${ }^{19}$ Yaiguaje v. Chevron Corporation, 2013 ONSC 2527.
} 
las cuales se solicitaba el exequátur a la luz de la doctrina del levantamiento del velo; concluyó en la ausencia de una relación directa y, con ello, en la falta de bienes de Chevron Corp. en Canadá. Dado que tampoco se aportaron pruebas que apuntaran de forma realista a que Chevron Corp. trasladará algunos de sus activos al país en un futuro cercano, el tribunal estimó que cualquier reconocimiento de la resolución de Ecuador carecería de efecto práctico y que, en cambio, conduciría a un gasto injustificado de tiempo, dinero y recursos judiciales. Por estos motivos optó por suspender el proceso de exequátur.

En apelación ${ }^{20}$, el tribunal superior analizó los fundamentos del inferior. Por lo que aquí interesa, concluyó que la exigencia de una "real and substantial connection between Ontario and the subject matter of the controversy" existe, pero solo cuando lo que se solicita es tutela por declaración, no cuando lo que se pide es tutela por reconocimiento. Ahora bien, también entendió que tal conclusión no es aplicable a Chevron Canada por cuanto ni había sido parte en el litigio en Ecuador ni había sido declarada responsable por la sentencia. La presencia de vínculos relevantes entre la empresa y el territorio, sumados a la significativa relación entre Chevron Corp. y Chevron Canada desde un punto de vista económico, permitieron al tribunal declararse competente, sin perjuicio no obstante del derecho de Chevron Canada de discutir que sus activos deban responder por la condena de Chevron Corp. en el estadio procedimental correspondiente.

A comienzos de abril de 2014 el Tribunal Supremo de Canadá accedió a la solicitud de Chevron de ser oída en relación con el procedimiento de reconocimiento en Ontario de la resolución de Lago Agrio ${ }^{21}$. La autorización otorgada a Chevron Corp. le permite concurrir a una vista y argumentar por qué el Tribunal Supremo debe revocar la decisión del tribunal de apelación de diciembre de 2013. Como es habitual, el Tribunal Supremo no acompañó su decisión de ninguna explicación.

\subsection{En Brasil}

La solicitud de exequátur de la decisión de Lago Agrio en Brasil se produjo en junio de 2012; en este momento sigue pendiente ${ }^{22}$. El órgano que debe pronunciarse acerca de ella es el Tribunal Supremo. En su defensa ante él, Chevron Corp. alegó argumentos relativos a la

\footnotetext{
2023 ONCA 758. Vínculo al pdf de la decisión: http://stevendonziger.com/2013/12/17/breaking-news-hugeappellate-victory-canada-ecuadorian-rainforest-communities/

${ }^{21}$ Que sepamos, la decisión no ha sido publicada aún.

${ }^{22}$ No hemos tenido acceso directo a esta decisión, como tampoco a las que se citan en relación con Argentina. El contenido de los fallos y los argumentos se reproducen en la doctrina consultada.
} 
corrupción y al comportamiento no profesional de los jueces en Ecuador y, además, cuestionó su independencia respecto a las interferencias políticas. Los alegatos podrían prosperar al amparo de la contrariedad al orden público, dependiendo de la amplitud con que el Tribunal Supremo interprete la condición examinada ${ }^{23}$.

Otros razonamientos de Chevron Corp. no se han suscitado nunca antes en el marco de un exequátur en Brasil, de ahí que cuál sea su validez resulte dudoso ${ }^{24}$. Así ocurre con la falta de competencia judicial de los tribunales de Brasil para decidir sobre el exequátur cuando la parte contra quien se solicita carece de vínculos significativos con el país. Chevron Corp. discute también la eficacia de la decisión de origen a la luz de las medidas adoptadas por el panel arbitral al amparo del BIT Ecuador-EE. UU.

\subsection{En Argentina}

Argentina ha sido otro de los países elegidos por los demandantes para solicitar la ejecución de la decisión de Lago Agrio. En realidad, a fin de evitar presumibles dificultades ligadas al exequátur de la decisión ecuatoriana y su colisión con una (generosa) excepción de orden público, la solicitud recayó sobre el embargo provisional decretado por la Corte de Sucumbíos de octubre de 2012, cuyo alcance se pretendía mundial. La petición, que debería hacerse efectiva contra cuatro compañías domiciliadas en Argentina - Chevron Argentina SRL, Ingeniero Priú SRL, CDC Aps y CDHC Aps-, se planteó además como simple solicitud de cooperación y como si la decisión final en Ecuador aún estuviera pendiente.

El exequátur fue efectivamente pronunciado a la luz de la Convención interamericana relativa a la ejecución de medidas cautelares, de 1979, que prevé también la denegación de la homologación por contrariedad al orden público, pero entendiendo este de forma más restrictiva que otros regímenes en vigor en Argentina. Chevron Corp recurrió la decisión en segunda instancia y luego en casación. Finalmente, el embargo fue levantado por el Tribunal Supremo el 4 de junio de 2013 sobre la base de la falta de identidad entre las entidades argentinas embargadas y Chevron Corp.: aquellas no habían sido partes en el proceso original y la ejecución subsecuente sobre sus activos no es posible so pena de violación de los artículos 17 y 18 de la Constitución de Argentina, que integran la cláusula de orden público internacional.

23 GÓMEZ, M. A., "The Global...", cit., p. 458. En pp. 458-459 el autor analiza la contrariedad del pronunciamiento en la parte relativa a los daños punitivos y el orden público; debe recordarse que esa parte fue revocada en casación (supra, bajo II, apartado 2).

${ }^{24}$ GÓMEZ M. A., “The Global...”, cit., pp. 456-457. 


\section{El arbitraje de inversiones}

\subsection{Las órdenes interinas "antiproceso"}

Con el telón de fondo del litigio entablado en Sucumbíos contra Chevron Corp. por los afectados por la contaminación, la compañía planteó demanda contra Ecuador por violación del BIT entre este país y EE. UU. en septiembre de $2009^{25}$. El arbitraje es administrado por la Corte Permanente de Arbitraje de La Haya y sigue las reglas UNCITRAL. En su reclamación, Chevron Corp. acusa a Ecuador de haber incumplido su obligación de "provide claimant's investment fair and equitable treatment, full protection and security, and treatement non less than that required by international law" y de garantizar al inversor "effective means of asserting claims and enforcing rights with respect to investment and investment agreements", y de discriminación. Más adelante, ya recaída la resolución de Lago Agrio, Chevron Corp. añadió denegación de justicia como consecuencia de una decisión judicial que habría sido pronunciada mediando fraude y corrupción, así como conspiración entre la República de Ecuador y los demandantes.

Hasta febrero de 2013 el panel arbitral dictó varias resoluciones interinas por las que se reclamaba a Ecuador la adopción de medidas para suspender el reconocimiento y/o la ejecución de la decisión ecuatoriana dentro y fuera del país. En particular, a través del segundo laudo interino relativo a medidas cautelares, de 16 de febrero de 2012, se ordenaba al demandado adoptar todas las medidas necesarias "whether by its judicial, legislative or executive branch" para suspender o provocar la suspensión del reconocimiento y la ejecución de la decisión de la Corte Provincial de Sucumbíos. En la fecha indicada el tribunal se pronunció declarando que la República de Ecuador ha violado las resoluciones previas ${ }^{26}$.

Ni el Tribunal Arbitral ni el estatal a quienes Ecuador solicitó la suspensión del proceso aceptaron los argumentos del país demandado ${ }^{27}$.

\subsection{La reacción de los particulares afectados}

\footnotetext{
${ }^{25}$ Chevron Corp. v. República de Ecuador, UNCITRAL Art., PCA caso núm. 2009-23.

${ }^{26}$ Ecuador se ha justificado alegando que su interferencia para paralizar las actividades de los demandantes en ejercicio de su tutela judicial efectiva sería ilegal e inconstitucional y crearía un conflicto de intereses entre Ecuador y sus nacionales.

${ }^{27}$ Sobre la negativa del Second Circuit, vid. 638 F.3d 384 (2d Cir. 2011). En relación con ella, es interesante el punto de vista de la doctrina según el cual la decisión de no suspender el proceso arbitral constituye una interferencia en el ejercicio de la soberanía de Ecuador: MA, D., "A BIT Unfair?: An Illustration of the Blacklash Against International Arbitration in Latin America”, 2012 J. Disp. Resol. 571, pp. 589-580. Según el mismo autor, la decisión de no paralizar el arbitraje redujo el interés de las partes por alcanzar un acuerdo (settlement) que habría puesto fin a la controversia.
} 
En febrero de 2009, como respuesta a la orden del Tribunal Arbitral, los demandantes en Lago Agrio solicitaron a su vez la adopción de medidas cautelares contra la República de Ecuador ante la Comisión Interamericana de Derechos Humanos. La petición se apoyaba en el riesgo que representaría para su derecho a compensación por daños al medio ambiente el que Ecuador paralizara la ejecución de la sentencia judicial - en cumplimiento, a su vez, de la decisión arbitral—. El otorgamiento de la medida habría sido posible al amparo del artículo 25 de las Reglas de Procedimiento de la Comisión. No obstante, la solicitud fue retirada; se especula acerca de los motivos ${ }^{28}$.

\subsection{Primer laudo arbitral parcial, track $I$}

A efectos del análisis de fondo, el Tribunal Arbitral ha separado dos vías o tracks en el proceso. La primera de ellas, la única abordada hasta el momento ${ }^{29}$, se centra exclusivamente en la interpretación del acuerdo de 1995 entre TexPet y Ecuador ${ }^{30}$ a fin de determinar la validez y el alcance de la renuncia de Ecuador a demandas futuras contra la empresa. En este sentido, el Tribunal ha concluido en un laudo parcial de 17 de septiembre de 2013 que: a) Chevron Corp., aun no siendo parte del citado acuerdo, también está afectado por él en calidad de beneficiario de la renuncia; b) Ecuador renunció en virtud del acuerdo al ejercicio de acciones colectivas, quedando incluidos también terceros; en cambio, no ha quedado afectado el derecho de acción de cada afectado singular por los daños individuales padecidos.

Quedan pendientes aún algunos puntos sobre interpretación de documentos, si bien probablemente no alterarán lo decidido en cuanto al sentido del acuerdo entre TexPet y Ecuador. El Tribunal tampoco se ha pronunciado aún (si bien se ha reservado la facultad y discreción de hacerlo posteriormente) a propósito del petitum de las partes, que comprende, entre otras cosas, la solicitud de Chevron Corp. de que se declare que la ejecución de la decisión de Lago Agrio dentro o fuera de Ecuador sería contraria a las obligaciones asumidas por la República bajo el acuerdo, el BIT y el derecho internacional, y de que se declare la nulidad de la sentencia de Lago Agrio a la luz del derecho internacional.

Según últimas informaciones, Ecuador ha solicitado la anulación del laudo y de las medidas interinas previas ante la Corte de Distrito de La Haya argumentando, entre otras cosas, la

\footnotetext{
${ }^{28}$ LOW, L., "Remarks", 106 Am. Soc'y Int'l L. Proc. 419 (2012), p. 421; GIORGETTI, C., "Mass Tort Claims in International Investment Proceedings: What Are the Lessons from the Ecuador-Chevron Dispute?", 34 U. Pa. J. Int'l L. 787 (2012-2013), pp. 792-793.

${ }^{29}$ La vista oral en el marco del track II debería haber tenido lugar en enero de 2014, pero Ecuador solicitó que fuera pospuesta.

${ }^{30}$ Vid. supra bajo II, apartado 1.
} 
violación del orden público; el mandato a Ecuador de suspender cautelarmente el proceso y, en su caso, la ejecución de la decisión de Lago Agrio contraviene el principio de independencia judicial y separación de poderes, en relación, además, con un proceso en el que el Estado no es parte. Se alega también que el Tribunal Arbitral ha actuado con exceso de poder al dictar un laudo tendente a privar a particulares del acceso a la justicia en defensa de su derecho a vivir en un medio ambiente sano.

\section{EL REPARTO DE ASUNTOS, O DÓNDE SE DISCUTE QUÉ}

\section{El argumento del fraude procesal: en origen o (¿y?) en el Estado requerido}

En los apartados anteriores hemos visto que el fraude procesal, en distintas manifestaciones que llegarían a implicar la corrupción de los jueces a cargo del caso de Lago Agrio, se configura como una de las defensas esenciales de Chevron Corp. en los escenarios en los que es demandada, y en particular en sede de solicitud de exequátur. Se entiende, además, que sería la clave de la defensa en los EE. UU. en caso de que el exequátur se intentara en este país. En este contexto se ha apuntado, sin embargo, una distinción de interés teórico y práctico por cuanto se refiere a la delimitación del alcance de las jurisdicciones nacionales en juego - la de origen, donde se pronuncia sentencia, y aquella en la que se solicita el exequátur- En efecto, es típico distinguir en EE. UU. (y se hacía también en Canadá) entre el fraude llamado extrínseco y el intrínseco. El primero se identifica con la conducta de una parte mediante la cual impide a la otra una defensa apropiada de su caso; por ejemplo, dirigiendo la notificación deliberadamente a una dirección incorrecta o proporcionando una información errónea sobre el lugar y el tiempo de la vista. El fraude intrínseco, en cambio, afecta al proceso mismo: se ponen como ejemplos un testimonio falso o la admisión de un documento falsificado. Solo el primer tipo de fraude es relevante a efectos de denegar el reconocimiento; la razón por la que se excluye el segundo radica en la voluntad de incentivar que el defecto se alegue en origen ante el mismo tribunal o el correspondiente superior - en todo caso, ante tribunales a los que el procedimiento discutido resulta familiar- 31 .

El fraude presuntamente cometido en el procedimiento en Sucumbíos reúne características de los dos tipos descritos, aunque predomina el fraude intrínseco. En estas circunstancias, se ha cuestionado la admisibilidad de la defensa de Chevron Corp. en EE. UU. sobre esta base, ya que aceptarla significaría un giro en la aplicación usual de la condición (cosa distinta es que

\footnotetext{
${ }^{31}$ WHYTOCK, C. A., "Some Cautionary Notes ...” cit. pp. 474-477.
} 
ello sea oportuno, habida cuenta de lo que está en juego y/o de la seriedad de los argumentos de Chevron $^{32}$ ).

En Canadá, la distinción entre fraude intrínseco y extrínseco ha perdido valor a efectos de exequátur; en su lugar se ha consagrado otra. Siendo conocidas las circunstancias que motivan el fraude, su alegación debe hacerse en origen en todo caso; una vez que el juez del fondo se ha pronunciado sobre este punto, su decisión es cosa juzgada. Solo cuando, aun mediando la debida diligencia, no hubiera sido posible detectar el fraude antes de la decisión original, cabrá alegarlo luego como motivo en contra del exequátur. Es por este motivo por lo que se entiende que la defensa de fraude (en caso de que se supere la fase actual y se pase a discutir sobre este punto) no sería útil a Chevron Corp. en Canadá, y ello porque se considera que en realidad la empresa ya la utilizó, aunque sin éxito, ante el juez de Lago Agrio ${ }^{33}$.

A nuestro entender, la idea de que la discusión sobre defectos de un sistema judicial como la corrupción, cuando afectan a sus operadores jurídicos más relevantes — los propios jueces-, debe tener lugar en su seno se comprende no solo a la luz de la mayor familiaridad o proximidad entre la jurisdicción y el supuesto, sino precisamente por razón de la cualidad que inviste a los sospechosos. Razones de cortesía internacional explican también que un Estado se cohíba a la hora de pronunciarse afirmando la corrupción como signo del sistema judicial de otro. No obstante, se impone realizar dos consideraciones: en primer lugar, es oportuno señalar lo paradójico de encomendar el examen de la corrupción judicial a órganos pertenecientes al mismo sistema, al menos cuando la tacha de corrupción se percibe como general; en segundo lugar, resulta difícil imaginar que consideraciones de cortesía primen sobre la no contrariedad con el orden público internacional en aquellos sistemas (que son la mayoría) en que este se erige en condición del reconocimiento/exequátur de las decisiones extranjeras. En este sentido, es importante poner de manifiesto que, si en principio el orden público internacional de cada país se nutre de concepciones propias, eventualmente distintas entre sí, en materia de corrupción hay un consenso sobre la necesidad de combatirla a todos los niveles y con todas las armas jurídicas, desde el derecho penal y también desde el civil, como atestiguan los convenios adoptados en el Consejo de Europa en 1999, y también a

\footnotetext{
${ }^{32}$ En este sentido, WHYTOCK, C. A., loc. ult. cit.; pero precisamente la excepcionalidad de las circunstancias conducen al autor a opinar que el cambio debe limitarse al supuesto concreto y no extenderse más allá.

${ }^{33}$ PENGELlEY, N., “We All Have Too Much Invested to Stop: Enforcing Chevron in Canada”, 2012, copia electrónica obtenida en SSRN (ulterior publicación en The advocate’s Quarterly).
} 
través de las posibilidades que ofrece el derecho internacional privado ${ }^{34}$. El acuerdo sobre este punto permite hablar de un orden público verdaderamente transnacional que justificaría volver a dar la oportunidad (con el límite del abuso) de alegar el fraude "intrínseco" en el Estado requerido, y que este rechace la decisión extranjera si lo entiende probado. Finalmente, aunque faltara ese consenso acerca de la corrupción como elemento de un orden público común, a nuestro entender es discutible que la alegación de corrupción sea desatendida y la resolución se reconozca cuando existan pruebas de esa corrupción con consecuencias sobre la posición procesal y la tutela judicial de una o de ambas partes; creemos que en ordenamientos como el nuestro lo proscribirían tanto el artículo 24 de la Constitución como el artículo $6 \mathrm{CEDH}$.

\section{Inversiones extranjeras y los derechos de terceros: ¿arbitraje o tutela judicial?}

La adopción de medidas "antiproceso" en el marco del arbitraje a fin de paralizar temporalmente procesos judiciales ante tribunales domésticos no es un fenómeno nuevo; tampoco es exclusivo del arbitraje de inversiones ${ }^{35}$. De acuerdo con un estudio doctrinal, entre 1972 y 2009 unos doce paneles de arbitraje ICSID han utilizado estas medidas con el objetivo de mantener el statu quo o, en algún caso, de defender la jurisdicción del Tribunal $\mathrm{Arbitral}^{36}$. Lo que es nuevo, y como tal lo reseñan (y critican) los autores, es la tendencia emergente en el arbitraje de inversiones a convertirse además en una instancia de apelación abierta a los inversores insatisfechos con las decisiones judiciales. De este modo, los árbitros pasan a ser "second-guessing judges, effectively vacating their rulings",37, que incluso han llegado a ordenar en el laudo definitivo poner fin a un proceso judicial ${ }^{38}$.

Conforme a lo anterior, de entrada la situación generada por el arbitraje en el caso Chevron Corp. c. República de Ecuador no es especial. Ciertamente, el Tribunal Arbitral de La Haya interfiere en el proceso judicial de Sucumbíos de varias formas. Por una parte, por la vía

\footnotetext{
${ }^{34}$ Vid. KRAMER, X., "Private International Law Responses to Corruption. Approaches to Jurisdiction and Foreign Judgments and the International Fight against Corruption", International Law and the Fight against Corruption, Asser Press, 2012, pp. 99-142.

${ }^{35}$ Vid. la cuestión prejudicial pendiente ante el TJUE, as. C- 536/13.

${ }^{36}$ GIL, R., "ICSID Provisional Measures to Enjoin Parallel Domestic Litigation", 2 Wordl Arb. \& Mediation Rev. 535 (2009), pp. 553-564.

${ }^{37}$ GOLDHABER, M.D., "The Rise of Arbitral Powers Over Domestic Courts", 1 Stan J. Complex Litig. 373 (2012-2013), pássim.

${ }^{38}$ ATA Construction, Industrial and Trading co. v. the Hashemite Kingdom of Jordan, ICSID Case $\mathrm{n}^{\circ}$ ARB/08/02, 18 de mayo de 2010, analizado por GOLDHABER, M.D., “The Rise...” cit., pp. 392-393.
} 
"tradicional" a través de las órdenes de suspensión cautelar de este. Por otra, de manera "novedosa", pronunciándose sobre una cuestión con implicaciones directas sobre el proceso judicial como es la de la existencia o no del derecho de acción colectiva y, junto con él, de la legitimación activa, con lo que los árbitros revisan además algo que ya había tratado el juez de Lago Agrio en su sentencia, concluyendo además de modo opuesto. Otras interferencias están aún en el aire pendientes de la decisión que en términos de "remedios" (relief) adopte el Tribunal Arbitral para completar el laudo parcial pronunciado en el track I.

Lo que resulta realmente llamativo en el caso ante el Tribunal Arbitral es que Ecuador - el demandado - no es parte en la litigación civil que se pretende parar: lo son los particulares damnificados ${ }^{39}$. En otras palabras, las partes en el procedimiento arbitral y en el judicial afectados no son las mismas. De ahí la pregunta acerca de la tutela judicial efectiva de los particulares a cuyo favor ha recaído la decisión de Lago Agrio, en Ecuador, pero también en otros Estados.

Las decisiones adoptadas en el marco del arbitraje de inversiones no limitan su alcance al ámbito geográfico y legal de Ecuador. Incluso aunque esta hubiera sido la intención de Chevron Corp., lo cierto es que son aptas para incidir en la acogida o no de la resolución de Lago Agrio en otros Estados. De entrada, el laudo podría ser causa de anulación de la sentencia ecuatoriana $^{40}$, que de este modo dejaría de existir; esta amenaza pesa sobre la decisión judicial y aconseja prudencia a la hora de otorgar su reconocimiento/exequátur. En segundo lugar, el laudo mismo puede ser un obstáculo a tal reconocimiento por incompatibilidad entre él y la sentencia judicial; llegado el caso, el Estado requerido debería elegir entre uno y otra de conformidad con el régimen en vigor, respetando en particular las obligaciones internacionales asumidas en el contexto del arbitraje de inversiones. En tercer lugar, lo que el laudo representa para el derecho a la tutela judicial efectiva de los particulares

\footnotetext{
${ }^{39}$ Se ha sostenido que el Estado sí estaría presente en el litigio civil en virtud de una (presunta) conspiración entre él y los demandantes: GOLDHABER, M.D., “The Rise...” cit., pp. 382-383, reproduciendo la respuesta de Chevron Corp. A nuestro juicio, aun cuando el dato de la conspiración fuera cierto, es discutible que valga como argumento suficiente; es decir, que determine una confusión de identidades entre aquellos demandantes y el Estado hasta el punto de eliminar la necesidad de intervención de los primeros en defensa de sus derechos e intereses en un proceso (el arbitral) que amenaza con revocar la decisión judicial recaída a su favor.

${ }^{40}$ Chevron Corp. solicita la nulidad conforme al derecho internacional y no al derecho procesal o sustantivo domésticos. Podría pensarse que el diferente origen del defecto imputado a la sentencia da lugar a consecuencias también diferentes. Creemos que para lo que a nosotros interesa no es así: la decisión no puede existir y no existir al mismo tiempo, sea cual sea la razón que provoca esa inexistencia.
} 
afectados, y, con ello, para su derecho material a un medio ambiente sano, apunta a la conveniencia de ignorarlo ${ }^{41}$.

En principio, la pregunta sobre el derecho a la tutela efectiva de los demandantes tiene una contestación inmediata en el carácter interno del problema; al fin y al cabo, fue Ecuador quien firmó con TexPet los acuerdos de renuncia a las acciones judiciales que los árbitros han interpretado en el sentido de que comprenden también a terceros. Si tal renuncia era posible y en qué términos, así como la valoración de sus consecuencias, deberá ser discutido por aquellos a quienes perjudica con los instrumentos que proporcione el propio sistema judicial; en su caso, ante instituciones internacionales de defensa de derechos humanos como la Comisión Interamericana o la Corte Interamericana. Presumiblemente, en estas instancias se llevará a cabo una ponderación entre los bienes sacrificados (el de tutela judicial de los individuos) y los valores o bienes favorecidos (la promoción de las inversiones y, a través de ellas y de sus beneficios para el país, el objetivo último del "bienestar general"42). Ello comportará un análisis de proporcionalidad de la limitación y un examen de las alternativas para los afectados, entre las que se encontraría la —inverosímil— posibilidad de acciones singulares, las cuales, si nos atenemos al laudo parcial, no han quedado excluidas por el acuerdo.

Cabe preguntarse si hay una alternativa a lo anterior mejor y más inmediata en cuanto a su puesta en práctica, y si lo sería el arbitraje de inversiones. Es en este contexto en el que se reaviva el debate sobre la transparencia, las garantías de imparcialidad de los árbitros, las posibilidades de apelación y, sobre todo, la apertura a terceros distintos de las partes iniciales. En circunstancias como la que subyace al entramado de Lago Agrio, es a todas luces esencial ofrecer a los terceros un acceso al Tribunal Arbitral y a ser oídos, ya sea vía amicus curiae, ya con una intervención en sentido propio ${ }^{43}$. Ahora bien, conforme a las reglas usuales en el ámbito del arbitraje de inversiones (las reglas UNCITRAL, ICC o ICSID son las típicas), estas posibilidades se encuentran muy limitadas en el momento actual; además, allí donde se ha admitido la intervención de terceros vía amicus curiae, el examen revela que el resultado

\footnotetext{
${ }^{41}$ Por supuesto, otros argumentos como el de corrupción de los jueces y fraude en el proceso continuarían afectando negativamente a las posibilidades de reconocimiento de la decisión ecuatoriana.

${ }^{42}$ Se señala incluso que el arbitraje de inversiones es bueno porque al reforzar la justicia para uno se refuerza (idealmente) la justicia para todos: GOLDHABER, M.D., “The Rise...” cit., p. 396.

43 KIMERLING, J., "Lessons from Chevron Ecuador Litigation: The Proposed Intervenor's Perspective", 1 Stan. J. Complex Litig. 241 (2012-2013).
} 
final no se ha visto afectado por ella ${ }^{44}$. Se añaden las dificultades para la articulación de la intervención de terceros en el marco del arbitraje de inversiones ${ }^{45}$ : la proverbial falta de transparencia en este marco dificulta el conocimiento necesario para articular la defensa; los particulares se verían involucrados en un sistema concebido a la luz de una filosofía específica para unos actores precisos (inversores y el Estado de inversión); y la incertidumbre sobre las reglas de procedimiento suma en detrimento de una defensa adecuada. Por otro lado, cuando lo que los individuos pretenden defender es su derecho fundamental a la tutela judicial efectiva, es legítimo pensar que la posibilidad de intervención no baste. Pasan a primer plano las dudas sobre la habilidad y la formación de los árbitros para llevar a cabo las operaciones de ponderación que necesariamente deben acompañar a las limitaciones de derechos fundamentales ${ }^{46}$.

En estas circunstancias, lo recomendable es prudencia por parte de los árbitros a la hora de ordenar la suspensión del proceso judicial, y permitir y fomentar la comunicación entre árbitros y jueces implicados.

\section{La falta de legitimación pasiva/falta de bienes: en exequátur o en fase de ejecución}

El embargo vinculado a la decisión de Lago Agrio fue revocado en Argentina en casación por falta de identidad entre el deudor condenado y las personas contra las cuales se solicitaba en exequátur. En Ontario, el argumento de la falta de bienes de Chevron Corp. en el territorio, canalizado por la vía de la independencia entre ella y su filial Chevron Canada, determinó la suspensión del proceso por falta de utilidad previsible de este ${ }^{47}$.

En otro lugar hemos defendido los beneficios de reconocer una decisión que no se puede ejecutar inmediatamente ${ }^{48}$. Cuestión distinta es que la falta de bienes sea un dato conocido por quien solicita el exequátur y que el objeto de pedirlo sea simplemente el de acosar al deudor; en estas circunstancias la petición debe ser rechazada por abuso de derecho. Pero, en todo caso, a nuestro juicio el razonamiento del tribunal de instancia de Ontario no prosperaría

\footnotetext{
${ }^{44}$ HARRISON, J., "Human Rights Arguments in Amicus Curiae Submissions: Promoting Social Justice", en Dupuy P.M. (ed.), Human Rights in International Investment Law and Arbitration, 2009, pp. 407-410.

45 Puede verse, entre otros muchos y además de la nota precedente: JACOB, M., "International Investment Agreements and Human Rights", INEF Research Paper Series, Human Rights, Corporate Responsibility and Sustainable Development, 03/2010; GOLDHABER, M.D., “The Rise...” cit., pp. 404-409.

${ }^{46}$ GOLDHABER, M.D., “The Rise...” cit., p. 413.

${ }^{47}$ Supra bajo III, apartado 1.2.

${ }^{48}$ Vid. supra, nota al pie núm. 1.
} 
en la fase de solicitud de reconocimiento/exequátur porque no es este el momento de discutir acerca de los bienes del deudor.

Con carácter general el procedimiento de reconocimiento/exequátur es breve y limitado a la verificación de condiciones precisas relativas al título exhibido por el acreedor; por ello, la identificación de los activos ejecutables, de no haberse realizado en la propia sentencia, procede con posterioridad en la fase de ejecución. Por las mismas razones relativas a lo expeditivo del proceso de exequátur, también es controvertida la oportunidad de solicitarlo contra quienes no han sido parte en el proceso inicial. El mero intento suscita la sospecha de violación del requisito relativo al respeto de los derechos de defensa de la persona contra quien se intenta. La suerte corrida por la petición de exequátur en Argentina ilustra lo que decimos. También lo hace el caso español: en la práctica se reiteran autos que apuntan a la conveniencia de plantear la solicitud de exequátur contra, exactamente, el mismo condenado en origen; la ausencia de identidad formal, sumada a lo limitado del procedimiento, condujo en todos ellos a una respuesta desfavorable a la petición de exequátur ${ }^{49}$. Lo limitado del procedimiento en que se decide el reconocimiento o se declara la ejecutabilidad de una resolución extranjera deja un margen muy escaso a argumentar de otro modo, en particular en términos de levantamiento del velo ${ }^{50}$.

La doctrina del levantamiento del velo societario es una reacción a la regla conforme a la cual los accionistas de una empresa de responsabilidad limitada no pueden ser considerados responsables por las deudas de esta, sumada al principio de la personalidad jurídica diferenciada de unos y otra; estas ideas se han trasladado también a los grupos de sociedades. En el escenario habitual de la litigación transnacional por daños al medio ambiente o a derechos humanos, lo que los demandantes suelen perseguir es la atribución de los actos de una filial a la empresa madre; el camino usual pasa por el levantamiento del velo. En la decisión de Lago Agrio el escenario es algo distinto; tal como se vio en Argentina y Canadá, el análisis se centra en la posibilidad de asociar activos de la filial a la empresa madre a fin de poder ejecutar sobre ellos la decisión recaída contra esta. El fenómeno se conoce

\footnotetext{
49 Autos de la Audiencia Provincial de Málaga de 22 de junio de 2000 (JUR $\backslash 2000 \backslash 283925$, fuente Westlaw Aranzadi); de 20 de septiembre de 2000 (JUR \2001\75705, fuente Westlaw Aranzadi); de 18 de octubre de 2000 (núm. 260/2000, JUR\2001\44012, fuente Westlaw Aranzadi); de 18 de octubre de 2000 (núm. 270/2000, JUR $\backslash 2001 \backslash 108636$, fuente Westlaw Aranzadi).

50 Vid., no obstante, el ATS de 24 de noviembre de 1998 (RJ $\backslash 1998 \backslash 9228$, fuente Westlaw Aranzadi), de reconocimiento de laudo arbitral, que rechaza la falta de legitimación pasiva en el procedimiento de exequátur y procede a levantar el velo bajo la cobertura de la verificación del respeto a los derechos de defensa (en el proceso de origen) de una de las sociedades en cuestión, lo que llevó a concluir que las entidades eran una sola.
} 
genéricamente como "reverse piercing of the veil"51. Aunque este dato es apto para generar una dificultad añadida, en primera instancia las construcciones ${ }^{52}$ para salvar el obstáculo representado por la separación de las empresas son igualmente aplicables, ya se trate de operar en un sentido (desde la filial hasta la empresa madre) o en otro (a fin de repercutir deudas de la empresa madre sobre la filial) ${ }^{53}$.

Las dificultades del levantamiento del velo son perfectamente conocidas; su grado varía según el sistema legal en juego (también, según la finalidad que se persiga a través de la operación $)^{54}$. De entrada, el intento ha de superar la convicción, más o menos arraigada, conforme a la cual la organización corporativa con la finalidad de eludir la responsabilidad es perfectamente legal ${ }^{55}$. El segundo obstáculo viene dado por la indefinición de los criterios o condiciones exigibles para alcanzar la convicción de la identidad entre las empresas concernidas; prácticamente todas las construcciones amparadas bajo la etiqueta de piercing of the veil son creaciones jurisprudenciales ${ }^{56}$ limitadas a los hechos del caso (fact-specific) y abiertas (open-ended).

Ante estos obstáculos, no es de extrañar que doctrina y práctica busquen otras soluciones; sin embargo, lo cierto es que su aplicación no es más sencilla ${ }^{57}$. Sobre la base de que el objetivo último del levantamiento del velo es promover la justicia y prevenir el fraude, se ha propuesto llevarlo a cabo sin más "when it is just and equitable to do so", lo que podría ocurrir

\footnotetext{
${ }^{51}$ La expresión es utilizada por el propio tribunal de Ontario en primera instancia. Un ejemplo célebre de "reverse piercing of the veil" es el caso inglés Amalgamated Investment \& Property Co. v. Texas Commercial Bank [1982] QB 84.

${ }^{52}$ En su mayoría doctrinales y jurisprudenciales. A nivel legislativo merece la pena recordar varias propuestas tendentes a imponer responsabilidad a corporaciones transnacionales vinculadas al territorio; así, el Bill for a Corporate Code of Conduct Act presentado ante la Cámara de Representantes de EE. UU. el 7 de junio de 2000; o el Corporate Code of Conduct Bill, de 6 de septiembre del mismo año, de Australia.

${ }^{53}$ No obstante, el levantamiento del velo es típicamente vertical y no horizontal, entendiendo por tal entre empresas "hermanas". Las condiciones que suelen requerirse al respecto (en particular el de control) se aplican con dificultad en el segundo escenario.

${ }^{54}$ Lo más habitual es la extensión de la responsabilidad, pero existen otras utilidades como, por ejemplo, establecer la competencia judicial internacional. En Canadá los solicitantes de exequátur se apoyan en la doctrina a fin de poder cobrar la condena con bienes de Chevron Canada; los demandados también, pero a fin de negar la identidad de las empresas y excluir con ello la competencia judicial del tribunal canadiense.

${ }^{55}$ Es el caso del RU, vid. Adams v. Cape [1990] 1 Ch. 433 (C.A.).

${ }^{56}$ Aunque hay situaciones específicas reguladas por ley en ámbitos concretos como la insolvencia.

${ }^{57}$ En el contexto de la litigación civil por daños medioambientales existe otra aproximación a la responsabilidad de la empresa madre que trata precisamente de eludir las dificultades del levantamiento del velo. Se trata de identificar un deber u obligación de la empresa madre cuyo incumplimiento está en el origen del daño y por el que es ella misma directamente responsable; vid., por todos, ENNEKING, L., Foreign Direct Liability and Beyond, Eleven International Publishing, 2012. El planteamiento no es útil en el contexto que aquí analizamos, en el que no se discute la responsabilidad de Chevron Corp., sino en qué consiste su patrimonio.
} 
precisamente en supuestos como el que subyace a la petición de exequátur en Canadá, Brasil y Argentina. De hecho, la posibilidad fue evocada por el tribunal de Ontario en la decisión de 1 de mayo de 2013, y rechazada por él. No es difícil entender este rechazo: basar el levantamiento del velo en la única condición —al mismo tiempo, objetivo - de promover la justicia y evitar el fraude es totalmente desaconsejable, pues resultaría sin duda en una praxis (aún) más impredecible que la del velo.

En otras propuestas se rechaza globalmente el principio de responsabilidad limitada en el seno de los grupos de sociedades a partir de la idea de que la dirección unitaria y la integración unitaria hacen del grupo una única empresa, una "distinct form of business association", abriendo el camino "a la evolución de un régimen jurídico especializado más allá del paradigma de la responsabilidad limitada de la sociedad por acciones" ${ }^{\text {"58 }}$. Desde esta perspectiva, la atribución de responsabilidad (y de derechos) resulta de la interrelación entre las empresas y no de su participación en los hechos de que se trate. Se alude a esta aproximación como "single economic unit doctrine" o "entreprise liability"59. En la práctica relacionada con daños medioambientales hay ejemplos de ella: se reconoce en el caso Amoco Cadiz, supuesto de naufragio de un petrolero $^{60}$, y se alude a ella en Bophal, donde el Gobierno indio aludió a la tesis como "sociedad transnacional monolítica ${ }^{61}$. En Europa una idea similar ha sido sugerida por la doctrina en términos de suspender por ley la separación de personalidad jurídica en casos específicos como son los relacionados con abusos de derechos humanos y daños al medio ambiente ${ }^{62}$.

\footnotetext{
${ }^{58}$ MUCHLINSKI, P.T., Multinational Enterprises and the Law, The Oxford International Library, 2007, p. 317. Por lo que respecta a la UE, ninguno de los ordenamientos examinados para el Study of the Legal Frame on Human Rights and the Environment Applicable to European Enterprises Operating Outside the European Union, encargado por la Comisión Europea a la Universidad de Edimburgo en 2010, reconoce explícitamente el concepto (vid. par. 190).

${ }^{59} \mathrm{Ph}$. BLUMBERG ha sido el gran defensor de esta concepción; vid., entre otros muchos, The Multinational Challenge to Corporation Law: The Search for a New Corporate Personality, Oxford University Press, 1993; "Accountability of Multinational Corporations: the Barriers Presented by Concepts of the Corporate Juridical Entity”, 24 Hastings Int'l \& Comp. L. Rev. 297 (2000-2001). En la actualidad está siendo retomada por otros autores; vid. DEARBORN, M., "Enterprise Liability: Reviewing and Revitalizing Liability for Corporate Groups", 97 California L. Rev. 195 (2009).

${ }^{60}$ Un resumen del razonamiento del tribunal puede encontrarse en SCOVAZZI, T., "Amoco Cadiz", La práctica internacional en materia de responsabilidad por accidentes industriales catastróficos, Valencia, 2005, pp. 2339, esp. pp. 23-27.

${ }^{61}$ In Re Union Carbide Corporation Gas Plant Disaster at Bophal, 634 F Supp 842 (SDNY 1986); para un análisis a fondo, vid. la obra colectiva (Baxi, U. y Paul, T., eds) Mass Disasters and Multinational Liability, N.M. Tripathi Private Ltd., Bombay, 1986.

${ }^{62}$ GREGOR, F. y ELLIS, H., "Fair Law: Legal Proposals to Improve Corporate Accountability for Environmental and Human Rights Abuses", ECCJ (European Coalition for Corporate Justice), Bruselas, 2008 pp. 13-14.
} 
La doctrina de la enterprise liability es sugerente; también es controvertida. Es en EE. UU. donde, aun con límites, ha tenido mayor aceptación ${ }^{63}$, mientras que es rechazada directamente en otras jurisdicciones como el Reino Unido; en ese mismo sentido se pronunció el juez de Ontario en la decisión de instancia relativa a la solicitud de exequátur. La práctica revela la misma incertidumbre, si no más, que la teoría del levantamiento del velo: conocer cuáles son los requisitos que dan lugar a la responsabilidad del grupo es igualmente confuso. Con certeza solo cabe decir que se sigue exigiendo una relación entre las empresas, normalmente en forma de control de una sobre la otra, y que otras condiciones típicamente asociadas al levantamiento del velo se suavizan y pierden importancia ${ }^{64}$.

A la vista de lo anterior, cabe concluir que lo aconsejable es solicitar el exequátur contra el deudor original y reservar la pretensión de extender la ejecución al patrimonio de otro (formalmente "otro") a la fase posterior de ejecución. En ese momento las condiciones del levantamiento del velo o similares doctrinas no son distintas, pero en general sí se dispone de más medios para ponerlas en práctica. En España, por ejemplo, la LEC prevé unos mecanismos de investigación en el patrimonio del deudor que pueden servir de ayuda al acreedor en ese momento, aunque no antes: por una parte, la obligación de hacer manifestación de bienes patrimoniales en cantidad suficiente para hacer frente a lo debido, sujeta a sanciones (art. 589 LEC); por otra, la posibilidad que se otorga al órgano judicial para dirigirse a las entidades financieras, los organismos y los registros públicos, así como a las personas físicas y jurídicas, que indique el acreedor a fin de que faciliten la relación de bienes del ejecutado (art. 590, art. 591).

\footnotetext{
${ }^{63}$ VANDEKERCKHOVE, K., Piercing the Corporte Veil, Wolters Kluwer, 2008, pp. 501-512, 531-532. La doctrina tiende a quedar restringida a marcos muy precisos; áreas particulares en las que la realización de una política legislativa de especial importancia estaría en peligro de admitirse la distinta identidad de los miembros del grupo. Un ejemplo en EE. UU. se produce precisamente en el ámbito de los daños al medio ambiente a través de la Comprehensive Environmental Response, Compensation and Liability Act de 1980 (CERCLA). Fuera de EE. UU. merece la pena citar el artículo 10 del Proyecto argentino de Código de Derecho Internacional Privado, en su versión de 1985, conforme al cual: "Multinational enterprises, such as conglomerates or holding companies, operating within the jurisdiction of several countries, despite the pluralistic nature of their legal personality, shall be considered economic units, and their activities shall be evaluated in the light of economic realities respecting their subjection to Argentinian law". La jurisprudencia doméstica de otros países ofrece ejemplos no etiquetados como "enterprise liability", pero que en la práctica corresponden con ella; vid. el amplio análisis de varios sistemas en VANDEKERCKHOVE, K., Piercing... cit., cap. 4.
}

${ }^{64}$ VANDEKERCKHOVE, K., Piercing... cit., p. 505. 


\section{CONCLUSIÓN}

La lección final de la saga de Lago Agrio es plural y trasciende al caso concreto. A día de hoy es el mejor ejemplo contemporáneo de la complejidad que generan las fronteras jurídicas y geográficas, de las oportunidades que derivan de su existencia y de los desafíos que plantean. Es también la mejor muestra de la necesidad de poner orden en el escenario de la litigación civil transnacional por daños al medio ambiente; la incorporación de forma abierta del ingrediente del arbitraje de inversiones no permite seguir posponiendo el problema de la relación entre este mecanismo de resolución de controversias y los procesos judiciales estatales.

Tras años de una batalla en la que todos han perdido, la peor parte se la llevan los damnificados; y en gran medida es dentro del sistema que debía protegerles donde se halla el origen de su mal. Los demandantes en Ecuador gozaron en varios foros del beneficio de la justicia gratuita; también tuvieron acceso a financiación por parte de terceros. Con ello se puede considerar salvado el desequilibrio de fuerzas que habitualmente se percibe entre las partes activa y pasiva en litigios similares al presente. Así las cosas, la verdadera asimetría deriva de la protección procesal incrementada de que goza el inversor extranjero, en un marco donde además el derecho de sociedades opera también a su favor. Dos datos que son habituales en el panorama de los movimientos económicos transfronterizos.

La decisión recaída en febrero de 2011 en Sucumbíos podría haber sido una sentencia ejemplar; el fallo contra Chevron Corp., un aviso con efectos disuasorios sobre las empresas multinacionales cuyas actividades en la industria extractiva en países de inversión se traducen en daños al medio ambiente y bienes asociados a él. Y, ciertamente, la litigación de Lago Agrio tiene un efecto disuasorio, si bien no es el buscado. Con independencia de que corresponda o no a la realidad, el mensaje que transmite acerca de Ecuador y de los (presuntos) déficits de su sistema judicial empaña la imagen del país y disminuye su atractivo para la inversión. El efecto negativo no se queda en las empresas y los inversores potenciales, también se extiende a la percepción de otros Estados. De facto, la solicitud de exequátur de la sentencia ecuatoriana en otros países va acompañada de una carga negativa, de una presunción (no legal) en contra. Y es que, en definitiva, la corrupción como medio para un fin no merece la pena, por muy legítimo que este fin sea. 


\section{BIBLIOGRAFÍA}

BLUMBERG, Ph., The Multinational Challenge to Corporation Law: The Search for a New Corporate Personality, Oxford University Press, 1993.

- "Accountability of Multinational Corporations: the Barriers Presented by Concepts of the Corporate Juridical Entity”, 24 Hastings Int'l \& Comp. L. Rev. 297 (2000-2001).

CASSELMEN B., "Chevron Expects to Fight Ecuador Lawsuit in US", Wall St. J., 20 de julio de 2009, aptdo. B3.

DEARBORN, M., "Enterprise Liability: Reviewing and Revitalizing Liability for Corporate Groups", 97 California L. Rev. 195 (2009).

DHOOGE L. J., "Aguinda v. Chevron Texaco: Discretionay Grounds for the Non-recognition of Foreign Judgments for Environmental Injury in the United States", 28 Va. Envtl. L.J. 241 (2010),

- "Aguinda v. Chevron Texaco: Mandatory Grounds for the Non-recognition of Foreign Judgments for Environmental Injury in the United States", 19 J. Transnat'l L. \& Pol'y 113 (2009-2010).

ENNEKING, L., Foreign Direct Liability and Beyond, Eleven International Publishing, 2012.

GIL, R., "ICSID Provisional Measures to Enjoin Parallel Domestic Litigation", 2 Wordl Arb. \& Mediation Rev. 535 (2009).

GIORGETTI, C., "Mass Tort Claims in International Investment Proceedings: What Are the Lessons from the Ecuador-Chevron Dispute?", 34 U. Pa. J. Int'l L. 787 (2012-2013).

GOLDHABER, M.D., "The Rise of Arbitral Powers Over Domestic Courts", 1 Stan J. Complex Litig. 373 (2012-2013).

GÓMEZ, M.A., "The Global Chase: Seeking the Recognition and Enforcement of the Lago Agrio Judgment Outside of Ecuador", 1 Stan. J. Complex. Litig. 429 (2012-2013).

GREGOR, F. y ELLIS, H., "Fair Law: Legal Proposals to Improve Corporate Accountability for Environmental and Human Rights Abuses", ECCJ (European Coalition for Corporate Justice), Bruselas, 2008.

HANSEN, R.F., "Multinational Enterprise Pursuit of Minimized Liability: Law, International Business Theory and the Prestige Oil Spill", 26 Berkeley J. Int'L L. 410.

HARRISON, J., "Human Rights Arguments in Amicus Curiae Submissions: Promoting Social Justice", Dupuy, P.M. (ed.), Human Rights in International Investment Law and Arbitration, 2009, pp. 407-410.

JACOB, M., "International Investment Agreements and Human Rights", INEF Research Paper Series, Human Rights, Corporate Responsibility and Sustainable Development, $03 / 2010$.

KIMERLING, J., 'Lessons from Chevron Ecuador Litigation: The Proposed Intervenor's Perspective", 1 Stan. J. Complex Litig. 241 (2012-2013).

KRAMER, X., "Private International Law Responses to Corruption. Approaches to Jurisdiction and Foreign Judgments and the International Fight against Corruption", International Law and the Fight against Corruption, Asser Press, 2012, pp. 99-142.

LOW, L., “Remarks”, 106 Am. Soc'y Int'l L. Proc. 419 (2012). 
MA, D., "A BIT Unfair? An Illustration of the Blacklash Against International Arbitration in Latin America", 2012 J. Disp. Resol. 571.

MUCHLINSKI, P.T., Multinational Enterprises and the Law, The Oxford International Library, 2007.

PATEL, S., "Delayed Justice: A Case Study of Texaco and the Republic of Ecuador's Operations, Harms, and Possible Redress in the Ecuadorian Amazon", 26 Tul. Envtl. L.J. 71 (2012).

PENGELLEY, N., "We All Have Too Much Invested to Stop: Enforcing Chevron in Canada", 2012 copia electrónica obtenida en SSRN (ulterior publicación en The advocate's Quarterly).

REQUEJO ISIDRO, M., "Aguinda v. Texaco: de EEUU a Ecuador, y vuelta (¿a ninguna parte?)", Pérez Carrillo, E., Empresa Responsable y Crecimiento Sostenible: Aspectos Conceptuales, Societarios y Financieros, ed. Thomson Reuter, 2012.

- "The Last Struggle for Redress: Recognition and Enforcement of Foreign Resolutions on Civil Liability for Human Rights Violations", Companies in Conflict Situations, ed. Generalitat de Catalunya/ICIP, 2013.

— “Aguinda v. Texaco en la fase de exequatur: análisis del primer tropiezo", Dereito, Revista Xurídica da Universidade de Santiago de Compostela, 2013.

SCOVAZZI, T., “Amoco Cadiz”, La práctica internacional en materia de responsabilidad por accidentes industriales catastróficos, Valencia, 2005.

SEIDERMAN, E., "The Recognition Act, Antisuit-Injunctions, the DJA, and Much More Fun: The Story of the Chevron-Ecuador Litigation and the Resulting Problems of Aggressive Mutinational Enforcement Proceedings", 41 Fordham Urb. L.J. 265 (2013).

STEINHARDT, R. G., “Remarks”, 106 Am. Soc'y Int'l L. Proc. 422 (2012).

Study of the Legal Frame on Human Rights and the Environment Applicable to European Enterprises Operating Outside the European Union, Universidad de Edimburgo, 2010.

VANDEKERCKHOVE, K., Piercing the Corporte Veil, Wolters Kluwer, 2008.

VV. AA. (Baxi, U. y Paul, T., eds.), Mass Disasters and Multinational Liability, N.M. Tripathi Private Ltd., Bombay, 1986.

WHYTOCK, C. A., "Some Cautionary Notes on the Chevronization of Transnational Litigation”, 1 Stan. J. Complex Litig. 467 (2012-2013).

- "The Chevron-Ecuador Case: Three Dimensions of Complexity in Transnational Dispute Resolution", 106 Am. Soc'y Int'l L. Proc. 425 (2012). 